\title{
Selective growth of gold nanostructures on locally amorphized silicon
}

\author{
Hiroki ITASAKA, Masayuki NISHI, ${ }^{\dagger}$ Yasuhiko SHIMOTSUMA, Kiyotaka MIURA, \\ Masashi WATANABE, ${ }^{*}$ Himanshu JAIN* and Kazuyuki HIRAO \\ Department of Material Chemistry, Graduate School of Engineering, Kyoto University, Nishikyo-ku, Kyoto 615-8510, Japan \\ *Department of Materials Science and Engineering, Lehigh University, 5 Packer Avenue, Bethlehem, PA 18015, USA
}

Locally selective formation of gold nanostructures on a silicon wafer without photolithography, electrolysis, or hydrofluoric acid was reported previously. With our previous method, gold nanostructures grow selectively on the locally amorphized surface on a silicon wafer when the silicon wafer is exposed to a customized Au ion-containing solution. The locally amorphized surface is produced by, for example, irradiation of a focused ion beam (FIB) or a femtosecond laser. The Au ion-containing solution is

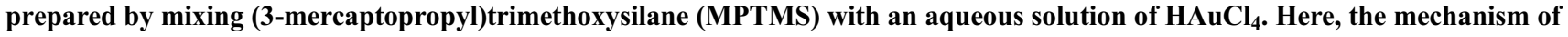
selective growth of gold nanostructures on the amorphized silicon is explained. Au ions in the solution placed on the FIB- or laserprocessed silicon are reduced by the amorphized silicon. Silicon-dangling bond defects, which exist in the amorphized silicon, seem to reduce the Au ions. Finally, we find that the use of MPTMS is unnecessary and a simple $\mathrm{HAuCl}_{4}$ aqueous solution produces desired results.

(C2014 The Ceramic Society of Japan. All rights reserved.

Key-words : Gold nanostructures, Silicon, Amorphous silicon, Selective growth, Danglingbond defects

[Received October 1, 2013; Accepted April 21, 2014]

\section{Introduction}

Gold nanostructures have attracted increasing attention because of their size-dependent properties such as localized surface plasmon resonance (LSPR), ${ }^{1}$ coulomb blockade, ${ }^{2)}$ and catalytic activity. ${ }^{3)}$ In order to utilize these properties efficiently, it is necessary to control the size and the integrated structure of gold nanostructures. Electron beam and UV lithographies are popular options for fabricating precise nanostructures. ${ }^{4), 5)}$ These techniques, however, require many steps due to their use of resist films for temporary-mask fabrication. A relatively simpler option for fabricating patterned gold nanostructures is maskless electrolytic deposition. ${ }^{6}$

In our previous work, ${ }^{7,8)}$ patterned gold nanostructures were successfully prepared on a crystalline silicon wafer with a maskless and electroless process as follows: an aqueous solution of hydrogen tetrachloroaurate $\left(\mathrm{HAuCl}_{4} \cdot 4 \mathrm{H}_{2} \mathrm{O}\right)$ was reacted with 3 -mercaptopropyltrimethoxysilane (MPTMS) at $100^{\circ} \mathrm{C}$. The product solution was dropped onto a silicon substrate surface that was previously exposed to a focused ion beam (FIB) or a femtosecond pulsed laser in preselected regions, followed by drying at $80^{\circ} \mathrm{C}$ in an electric furnace. MPTMS functioned as a reducing agent for $\mathrm{Au}$ (III) ions to $\mathrm{Au}$ (I) ions in the solution and did not work as a coupling agent. Hydrofluoric acid, which is commonly used for electroless methods, ${ }^{9)}$ was unnecessary for our method. MicroRaman spectroscopy revealed that amorphization of silicon was induced by FIB or femtosecond laser irradiations when gold nanostructures successfully grew on the irradiated area; therefore, the amorphization of silicon was the first crucial step for the growth of gold nanostructures. However, the details of the growth mechanism have remained unclear so far.

$\uparrow$ Corresponding author: M. Nishi; E-mail: west@collon1.kuic. kyoto-u.ac.jp
In the present work, we pursue two basic questions concerning the role of the amorphized silicon in the gold-nanostructure growth: (1) Do gold nanostructures grow on the preprocessed surface before drying or during drying? (2) What is it in the amorphized silicon, but not in crystalline silicon, that leads to the growth of gold nanostructures? To answer the first question, the silicon substrate, on which a drop of $\mathrm{Au}$ ion-containing solution has been placed, is rinsed with ethanol before drying. If gold nanostructures are observed on the preprocessed area, they should have grown before drying. To answer the second question, we focus on dangling-bond defects, which exist in much higher concentration in the amorphized silicon than in crystalline silicon. Since the dangling-bond defects are reactive and easily oxidize, the extent of growth of gold nanostructures should depend on the surface oxidation of the amorphized silicon. To investigate such correlation, a sputter-deposited amorphous silicon thin film, instead of locally amorphized silicon, is used as the test surface. An amorphous silicon thin film can be easily produced by sputtering in vacuum, and the film is wide enough for the spatial resolution of a conventional X-ray photoelectron spectroscopy (XPS) equipment, which is an effective tool for evaluating surface oxidation. Therefore, a thin film of the sputtered amorphous silicon fits our present purpose. For comparison, fracture surfaces of a crystalline silicon substrate, which also have dangling bonds, are subjected both to the gold growth procedure and XPS analysis.

\section{Experiment}

To investigate whether gold nanostructures grew on the preprocessed area before or during drying, the silicon substrate, on which a drop of Au ion-containing solution was placed, was rinsed with ethanol before drying as follows. Undoped Si (100) wafer was cut into $5 \mathrm{~mm}$ squares and pre-cleaned by sonication for $5 \mathrm{~min}$ in ethanol. A preselected region of the substrate was irradiated with a $1 \mathrm{kHz}$ amplified fiber laser (Ifrit, Cyber Laser), 
which delivers pulses with a duration of $238 \mathrm{fs}$ and a center wavelength of $780 \mathrm{~nm}$. The laser beam was focused with an objective lens, and its energy fluence $\left(2 \mathrm{E} / \pi \omega_{0}^{2}\right)$ was $2.8 \mathrm{~J} / \mathrm{cm}^{2}$. A suspension, which was prepared by stirring a mixture of $17.5 \mu \mathrm{l}$ of 2 -mercaptoethanol and $3 \mathrm{ml}$ of $0.05 \mathrm{M} \mathrm{HAuCl}_{4}$ aq. on a hot plate set to $100^{\circ} \mathrm{C}$ for $10 \mathrm{~min}$, was then dropped onto the substrate. Here, 2-mercaptoethanol was used instead of MPTMS that was used in our previous study. Five minutes later, the substrate was rinsed with ethanol and dried in air at room temperature. The surface of the substrate was observed using an FE-SEM (JSM6700F, JEOL, Japan).

We followed the following procedure to investigate whether or not the gold-nanostructure growth depends on the degree of oxidation of the silicon surface. Amorphous silicon thin films with the thickness of about $100 \mathrm{~nm}$ were prepared on a crystalline silicon substrate by radio frequency spattering (SPF-332H, ANELVA) at room temperature. The sputtering deposition was carried out with an rf power of $50 \mathrm{~W}$ at a sputter gas pressure of 0.3 Pa under pure Ar gas. The thickness of the sputtered thin film was measured by Alpha-Step IQ (KLA-Tencor). The amorphous nature of silicon film was identified by X-ray diffraction (XRD) analysis. The obtained thin films were stored in a desiccator for 2-432 h. Then, the Au ion-containing suspension described above was dropped onto the thin films. One minute later, the thin films were rinsed with ethanol and then dried in air. Fracture surfaces of crystalline silicon were also exposed to the same procedure. To evaluate the degree of oxidation of the silicon surfaces, XPS Si 2p spectrum was measured by using $\mathrm{Mg} \mathrm{K} \alpha$ excitation (MT-5500, ULVAC-PHI, Inc.). ${ }^{10), 11)}$

\section{Results and discussion}

Figure 1 shows SEM images of the femtosecond-laserirradiated area after the gold-growth procedure that (a) includes the rinse with ethanol before drying at room temperature, or (b) excludes the rinse but includes drying at $80^{\circ} \mathrm{C}$. As can be seen, gold nanostructures selectively grew on the laser-irradiated area whether the gold-growth procedure included the ethanol rinse or not. This result proves that gold nanostructures grow before drying.

The rinse procedure gave us a new finding: even if a simple $\mathrm{HAuCl}_{4}$ aqueous solution is used, the gold nanostructures successfully grow on the preprocessed area. Figure 2 shows an SEM image of a prepared pattern composed of gold nanostructures that grew from a simple $\mathrm{HAuCl}_{4}$ aqueous solution on the FIB-amorphized silicon surface. All procedures that included rinsing and drying were performed at room temperature. These results revealed that FIB- or femtosecond-laser-induced amorphous silicon reduced Au ions to gold nanostructures.

MPTMS, which appeared necessary in our previous non-rinse procedure, ${ }^{7)}$ is not required in fact. The reason can be as follows:
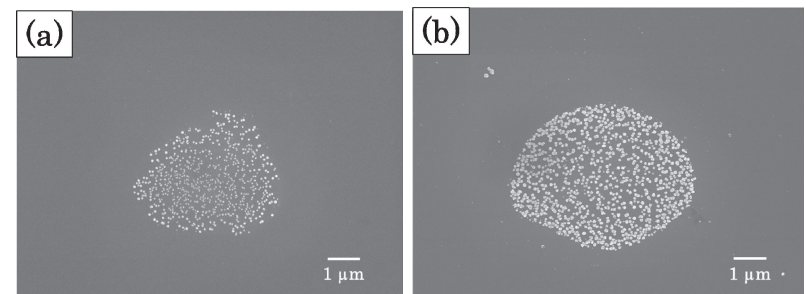

Fig. 1. SEM images of the femtosecond-laser-irradiated area after the gold-growth procedure that (a) includes the rinse with ethanol before drying at room temperature, or (b) excludes the rinse but includes drying at $80^{\circ} \mathrm{C}$.
If a $\mathrm{HAuCl}_{4}$ aqueous solution is used in the previous non-rinse procedure, a gold chloride thin layer forms on the substrate under drying due to concentration, and prevents us from identifying the gold nanostructures by SEM observation. Conversely, the thin layer formation is avoided when MPTMS is added. MPTMSderived small impurity particles, which form via hydrolytic condensation when MPTMS is mixed into $\mathrm{HAuCl}_{4}$ aqueous solution, can remove their surrounding solution on the substrate under drying, and enable us to identify the gold nanostructures by SEM observation. Rinse procedure before drying can also remove the solution on the substrate.

The amorphized silicon, which can reduce $\mathrm{Au}$ ions to gold nanostructures as shown and discussed above, commonly has much more dangling-bond defects than crystalline silicon. These defects, which are energetically located at around the Fermi level of the intrinsic silicon, seem to reduce Au ions. They are reactive and easily oxidize. Therefore, gold-nanostructure growth should depend on the oxidation of the amorphized silicon surface. Figures 3(a)-3(d) show SEM images of gold nanostructures that grew on the sputter-deposited amorphous-silicon thin films stored in a desiccator for $2-432 \mathrm{~h}$ after the thin-film preparation. The sputter-deposited amorphous silicon was used here instead of FIB- or femtosecond-laser-induced amorphous silicon due to the limitation in spatial resolution of available XPS equipment. As can be seen, gold nanostructures grew on each surface of the sputter-deposited amorphous silicon, and their number decreased with the increasing storage time of the as-sputter-deposited thin film in the desiccator. Figure 3(e) shows Si 2p XPS spectra of the surfaces of the as-sputter-deposited thin films stored in a desiccator. The intensity ratio of the peak at $\sim 102-103 \mathrm{eV}$ to that at $\sim 99 \mathrm{eV}$ increased with the increasing storage time. The former and latter peaks indicate silicon oxide ( $\mathrm{SiOx}$ ) and silicon, respectively. ${ }^{10), 11)}$ These results correlate very well to the reduction of the number of gold nanostructures with oxidation of the amorphous silicon surface. Deactivation of dangling-bond defects by surface oxidation $^{12), 13)}$ can be one reason for the decrease in the number of gold nanostructures.

The surface of freshly fractured crystalline silicon has danglingbond defects like the amorphized silicon. Figure 4 shows SEM images of gold nanostructures that grew on the fracture surface of a crystalline silicon substrate stored in the desiccator for (a) $5 \mathrm{~min}$ and (b) $24 \mathrm{~h}$ after the silicon substrate was fractured. As in the case of the sputter-deposited amorphous-silicon surface, gold nanostructures grew on each surface of the fracture surface, and their number decreased with the increasing storage time in the

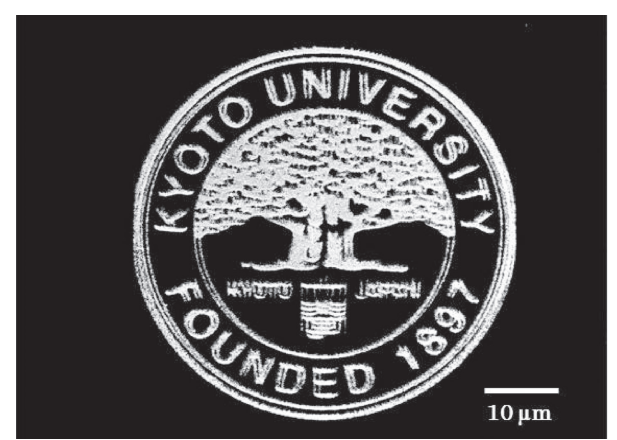

Fig. 2. An SEM image of a prepared pattern composed of gold nanostructures that grew from a simple $\mathrm{HAuCl}_{4}$ aqueous solution on the FIB-amorphized silicon surface. The substrate, on which the $\mathrm{HAuCl}_{4}$ aqueous solution was dropped, was rinsed with ethanol before drying. All procedures were performed at room temperature. 

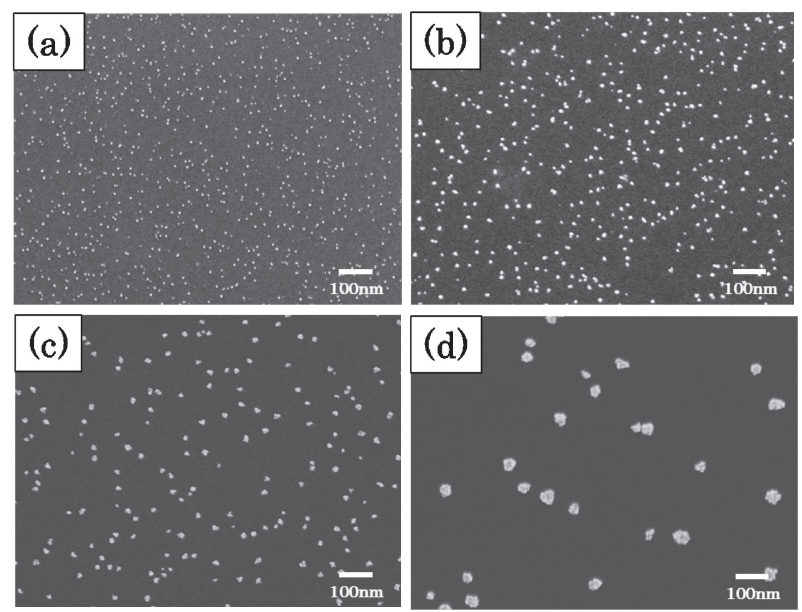

(e)

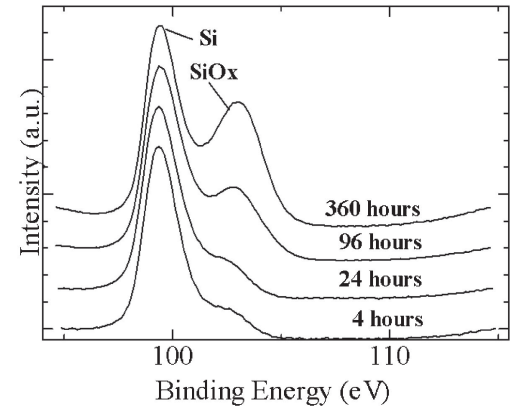

Fig. 3. SEM images of gold nanostructures that grew on the sputterdeposited amorphous-silicon thin film stored in a desiccator for (a) $8 \mathrm{~min}$, (b) $24 \mathrm{~h}$, (c) $48 \mathrm{~h}$, and (d) $96 \mathrm{~h}$ after the thin-film preparation. Si 2p XPS spectra of the surfaces of the amorphous-silicon thin films stored in a desiccator were shown in (e). The spectra are normalized at $99 \mathrm{eV}$.
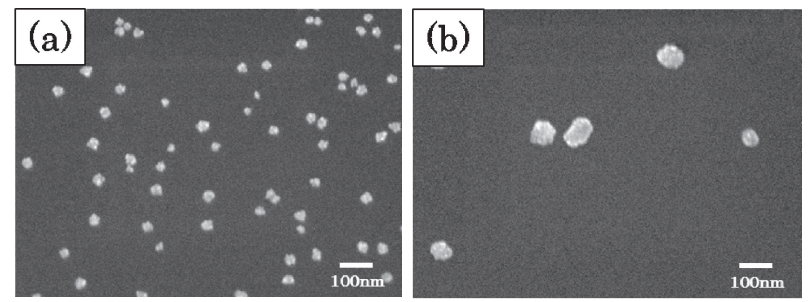

Fig. 4. SEM images of gold nanostructures that grew on a fracture surface of crystalline silicon substrate stored in a desiccator for (a) $5 \mathrm{~min}$ and (b) $24 \mathrm{~h}$ after the substrate was fractured. desiccator. The fracture surface of the crystalline silicon substrate, however, had a much smaller number of gold nanostructures than the sputter-deposited amorphous-silicon surface at the same storage time in the desiccator.

The numbers of gold nanostructures that grew on the sputterdeposited amorphous-silicon surface and the fracture surface of crystalline silicon were quantitatively measured by image processing of SEM images using Igor 5. Figure 5 shows (a) the number of gold nanostructures per unit area and (b) the area ratio of gold to silicon as a function of the storage time of the sputter-deposited and the fractured silicon in the desiccator. On the fracture surface of crystalline silicon, both the number per unit area and the area ratio of the gold nanostructures decreased more rapidly with the increasing storage time than those on the sputter-deposited amorphous-silicon surface. Figure 6 shows the degrees of surface-silicon oxidation of the sputter-deposited amorphous silicon surface and the fracture surface of crystalline silicon as a function of the storage time in the desiccator after the preparation of the target silicon surfaces. The degree of oxidation is here defined as $A_{\mathrm{SiOx}} /\left(\mathrm{A}_{\mathrm{Si}}+\mathrm{A}_{\mathrm{SiOx}}\right)$, where $\mathrm{A}_{\mathrm{Si}}$ and $\mathrm{A}_{\mathrm{SiOx}}$ are the areas of Si $2 p$ XPS peaks assigned to silicon and oxidized silicon, respectively. It is worth mentioning that XPS provides the information from the top $3 \mathrm{~nm}$ of silicon surface under the present experimental conditions. ${ }^{14), 15)}$ Both surfaces rapidly oxidized

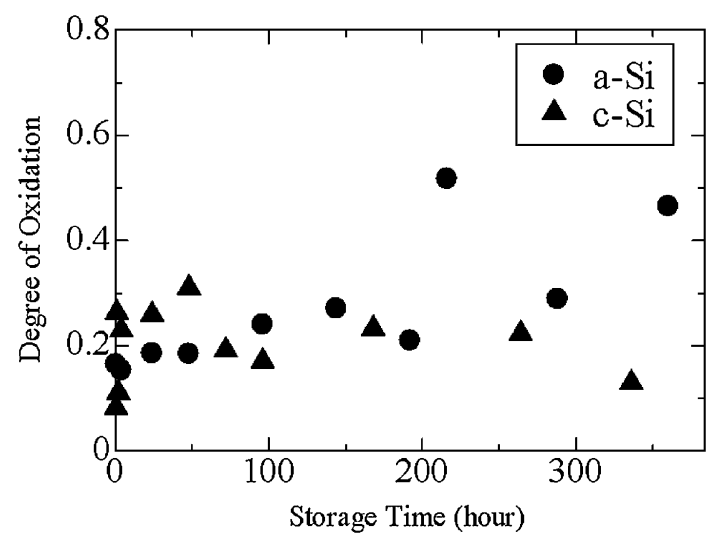

Fig. 6. The degrees of surface-silicon oxidation of the sputter-deposited amorphous-silicon surface and the fracture surface of crystalline silicon as a function of the storage time in the desiccator. The degree of oxidation is defined as $\mathrm{A}_{\mathrm{SiOx}} /\left(\mathrm{A}_{\mathrm{Si}}+\mathrm{A}_{\mathrm{SiOX}}\right)$, where $\mathrm{A}_{\mathrm{Si}}$ and $\mathrm{A}_{\mathrm{SiOx}}$ are the areas of Si $2 \mathrm{p}$ XPS peaks assigned to silicon and oxidized silicon, respectively.
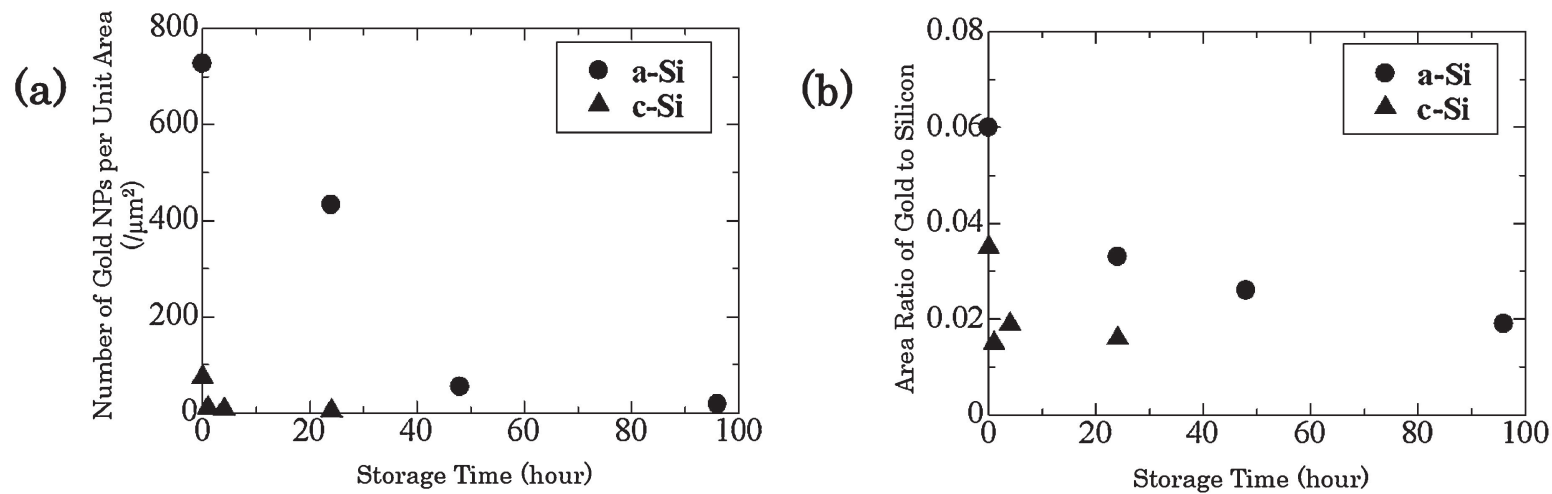

Fig. 5. Results of SEM-image processing of the gold nanostructures that grew on the sputter-deposited amorphous-silicon surface and the fracture surface of crystalline silicon: (a) the number of gold nanostructures per unit area and (b) the area ratio of gold to silicon as a function of the storage time of the sputter-deposited and the fractured silicon in the desiccator. 
( $\sim 20 \%$ of the XPS-detectable silicon) after the preparation of the target silicon surfaces. After the rapid oxidation, the sputterdeposited amorphous-silicon surface slowly continued to oxidize, and the fracture surface of crystalline silicon does not seem to oxidize further during the present experimental time scale. This difference can be caused by the following fact: The danglingbond defects exist only at the outermost surface of the fractured crystalline silicon. In contrast, the dangling-bond defects exist everywhere in the sputter-deposited amorphous silicon with a thickness of $100 \mathrm{~nm}$. Slow oxidation observed only for the sputter-deposited amorphous silicon is attributable to the dangling-bond defects in the surface volume inside the outermost atomic surface layer. The dangling-bond defects at the outermost layer can cause the rapid oxidation observed for both the sputterdeposited silicon and the fractured crystalline silicon. The dangling-bond defects that can induce the further slow oxidation for the sputter-deposited silicon can also induce the larger number of gold nanostructures than those on the fracture surface of crystalline silicon. These dangling-bond defects slowly oxidize in air, but rapidly reduce $\mathrm{Au}$ ions when exposed to an $\mathrm{Au}$ ioncontaining solution. The discussion of size or volume of gold nanostructures is beyond the scope of the present work, since, once $\mathrm{Au}-\mathrm{Si}$ interface forms, the electrons in silicon may reduce $\mathrm{Au}$ ions on the gold surface across the Au-Si interface owing to the fact that the junction of Au-Si is Schottky contact and the Fermi level of gold is higher than that of the Au ion-containing solution. Future work will reveal the details.

\section{Conclusions}

Gold nanostructures selectively grew on the FIB-amorphized silicon surface on a crystalline silicon wafer when exposed to a simple $\mathrm{HAuCl}_{4}$ aqueous solution. MPTMS, which was considered necessary in our previous report, is not required; but the present method includes a new rinse step to the previous method. The $\mathrm{Au}$ ions in the solution that were dropped onto the FIBprocessed silicon are reduced by the FIB-amorphized silicon, resulting in the growth of gold nanostructures locally and selectively. The dangling-bond defects, which are energetically located at around the Fermi level and exist in large concentration in the amorphized silicon, seem to reduce the Au ions. Locally producing non-oxidized amorphous silicon surface on a naturally oxidized crystalline silicon surface is the key to the local selective growth of gold nanostructures on a silicon wafer.

Acknowledgements This research was supported in part by the Global COE Program "International Center for Integrated Research and Advanced Education in Materials Science" (No. B-09) and KAKENHI Grant Number 25288107 by the Japan Society for the Promotion of Science. We also thank the National Science Foundation for initiating and supporting our international collaboration through the International Materials Institute for New Functionality in Glass (IMI-NFG: NSF Grant No. DMR-0844014).

\section{Referencesz}

1) M. A. El-Sayed, Acc. Chem. Res., 34, 257-264 (2001).

2) R. Parthasarathy, X.-M. Lin and H. M. Jaeger, Phys. Rev. Lett., 87, 186807 (2001).

3) M. Haruta, N. Yamada, T. Kobayashi and S. Iijima, J. Catal., 115, 301-309 (1989)

4) A. Gopinath, S. V. Boriskina, B. M. Reinhard and L. D. Negro, Opt. Express, 17, 3741-3753 (2009).

5) L. Rosa, K. Sun, J. Szymanska, F. E. Hudson, A. Dzurak, A. Linden, S. Bauerdick, L. Peto and S. Juodkazis, Phys. Status Solidi RRL, 4, 262-264 (2010).

6) P. Schmuki, L. E. Erickson and G. Champion, J. Electrochem. Soc., 148, C177-C182 (2001).

7) T. Matsuoka, M. Nishi, Y. Shimotsuma, K. Miura and K. Hirao, J. Ceram. Soc. Japan, 118, 575-578 (2010).

8) T. Matsuoka, M. Nishi, Y. Shimotsuma, K. Miura and K. Hirao, IOP Conf. Ser: Mater. Sci. Eng., 18, 052007 (2011).

9) N. Kubo, T. Homma, Y. Hondo and T. Osaka, Electrochem. Acta, 51, 834-837 (2003).

10) A. Yoshigoe, K. Moritani and Y. Teraoka, Appl. Surf. Sci., 216, 388-394 (2003).

11) A. Namiki, K. Tanimoto, T. Nakamura, N. Ohtake and $T$. Suzuki, Surf. Sci., 222, 530-554 (1989).

12) B. Schubert, P. Avouris and R. Hoffmann, J. Chem. Phys., 98, 7593-7605 (1993).

13) $\mathrm{Ph}$. Avouris and D. Cahill, Ultramicroscopy, 42-44, 838-844 (1992).

14) S. Tanuma, C. J. Powell and D. R. Penn, Surf. Interface Anal., 17, 911-926 (1991).

15) S. Tanuma, C. J. Powell and D. R. Penn, Surf. Interface Anal., 17, 927-939 (1991). 